\title{
Survey of the human acetylator polymorphism in spontaneous disorders
}

\author{
D A PRICE EVANS
}

From the Department of Medicine, Riyadh Armed Forces Hospital, PO Box 7897, Riyadh 11159, Kingdom of Saudi Arabia.

SUMMARY There is ample evidence that the human acetylator phenotypes are associated with drug induced phenomena. It is principally the slow acetylators who exhibit toxic adverse effects because of their relative inability to detoxify the original drug compounds. In rare instances, however, it is the rapid acetylators who are at a disadvantage.

In the matter of association of spontaneous disease with either acetylator phenotype, there are two groups of disorders to consider. First, disorders in which carcinogenic amines are known to be an aetiological factor. This is because these amines are substrates for the polymorphic $\mathrm{N}$-acetyltransferase activity and hence there is a possible rational basis for searching for an association. Secondly, other disorders where searches for associations are based more on hunches.

In the first group there is a definite statistical association between cancer of the bladder and the slow acetylator phenotype. In prevalence studies the slow phenotype is $39 \%$ more asscciated with bladder cancer than is the rapid phenotype. On the basis of the evidence now available it is not possible to say whether this association is because slow acetylators develop the disease more frequently or whether they survive longer. In the second group the relevant studies show (1) a greatly increased prevalence of slow acetylators in Gilbert's disease; (2) a confirmed association between the rapid acetylator phenotype and diabetes; (3) a possible association between the rapid acetylator phenotype and breast cancer; (4) a possible association between the slow acetylator phenotype and leprosy in Chinese patients; (5) an earlier age of onset of thyrotoxicosis (Graves' disease) in slow acetylators than in rapid acetylators; (6) no evidence of an association between either phenotype and spontaneous systemic lupus erythematosus.

There are many published studies on the relationship of the acetylator polymorphism to drug induced disorders. Slow acetylators exhibit toxic reactions to the original unchanged drug compound more frequently than rapid acetylators. In a few clinical situations, however, rapid acetylators fare worse than slow acetylators. A number of publications describe surveys of the acetylator phenotypes in patients with spontaneous disorders. The objective of this article is to evaluate these studies.

\section{Methods}

POINTS WHICH HAVE TO BE CONSIDERED IN STUDIES OF ASSOCIATIONS BETWEEN THE ACETYLATOR POLYMORPHISM PHENOTYPES AND SPONTANEOUS DISORDERS

(1) Was the disease entity being investigated adequately defined?

On the whole it is preferable to have the disease Received for publication 30 September 1983. Accepted for publication 23 November 1983. entity defined by some internationally accepted criterion, for example, systemic lupus erythematosus (SLE) as defined by the American Rheumatological Association. ${ }^{1}$ There was very little value in, for example, publishing the phenotypes of 100 patients with various lung disorders without providing details of the diagnoses. ${ }^{2}$

(2) Was the ethnic composition of both patient and control groups defined?

It is essential to know the ethnic composition of the patient and control groups. For example, in the otherwise excellent paper of Zacest and KochWeser, ${ }^{3}$ the ethnic composition of the group of 20 hypertensives studied in Boston, Massachusetts is not stated. Sometimes an arbitrary assumption with regard to ethnic group seems warranted, for example, presumably Lawson et $a l^{4}$ were studying SLE and rheumatoid arthritis in white Glasgow citizens. 
(3) Was the patient sample a stratum (that is, derived from a selected group within the population)?

It is preferable to have as unselected a sample of the disorder being investigated as possible. Sometimes, however, a selection is inevitable, for example, it is possible that a particularly severely afflicted group is studied simply because they attend a special hospital clinic for the disorder under consideration.

(4) Was the phenotyping test performed in a technically satisfactory manner, that is, was the phenotype definition satisfactory?

It is clear that ambiguous results (subjects with 'intermediate' results) are best avoided by rigorous attention to detail. For example, when using the sulphamethazine test of Evans, ${ }^{5}$ some investigators omit the collection of the blood sample and rely solely on the urine specimen. Obviously the same confidence cannot be placed in the results as if both specimens were collected and their results demonstrated to be in agreement with each other in the form of a scattergram. The separation between the two phenotypes must be clear. When a diagram is constructed from the data in Godeau et al, ${ }^{6}$ for example, it is not possible to assess the phenotype frequency in their systemic lupus erythematosus patients, so this study is not considered further.

(5) Did the disease condition being investigated interfere with the phenotyping test?

Since the sulphadimidine phenotyping test of Evans ${ }^{5}$ depends upon renal and hepatic function, disorders of the kidney and liver could interfere with the results obtained.

Fine and Sumner, ${ }^{7}$ who studied 10 uraemic patients, state that there is a relatively greater retention of acetyl sulphadimidine in uraemia. This could influence the phenotyping procedure by producing an increased ratio of acetylated drug in the blood and a diminished ratio in the urine. Furthermore, the acetylation process is induced in severe and prolonged uraemia. Hall, ${ }^{8}$ however, gives evidence from phenotyping 57 patients with varying degrees of diminished creatinine clearance, and his scattergram shows two distinct groups, viz slow and rapid acetylators. (There is a difference between absolute values and the overall pattern, and it is the latter which gives the phenotyping.)

With regard to liver disorders, Levi et al ${ }^{9}$ showed in 29 subjects with serum bilirubin concentrations less than $2 \mathrm{mg} / 100 \mathrm{ml}$ that isoniazid half life distributions show a clear bimodal curve enabling phenotype recognition as in healthy subjects. In 39 subjects with serum bilirubin concentrations greater than $2 \mathrm{mg} / 100 \mathrm{ml}$ the antimode is so blurred that phenotype recognition is not possible.
A group of patients with potential liver damage is alcoholics. Lester ${ }^{10}$ showed isoniazid half life data on 28 alcoholics (without ethanol in their blood) and the distribution would appear to be bimodal as in healthy subjects. However, he found that maintenance of 0.02 to $0.04 \%$ ethanol in the blood before and during the determination of the half lives resulted in a $30 \%$ decrease in the half lives in both groups of subjects. Similarly, Olsen and Morland ${ }^{11}$ showed that the presence of about $1 \mathrm{~g} / \mathrm{l}$ of ethanol in the blood decreased the apparent half life of sulphadimidine by about $20 \%$ in both acetylator phenotypes. Olsen and Morland ${ }^{12}$ showed that ethanol causes a significant reduction in the plasma half life of procainamide with an increase in total clearance. This effect occurs in both phenotypes. The volume of distribution was unaffected.

The position and activity of the subjects being phenotyped may be of importance. For example, Levi et $a l^{9}$ point out that isoniazid half lives in patients with liver disease are increased by about $20 \%$ by the change from lying to walking. On the other hand, pretreatment with other drugs (mainly with barbiturates) has no effect on the distribution curve of isoniazid half lives in normal subjects.

An increased acetylation of sulphadiazine of six undernourished as compared with six well nourished subjects was demonstrated by Shastri and Krishnaswamy ${ }^{13}$ In a further investigation, ${ }^{14}$ however, undernutrition was found not to alter the acetylator phenotype of male adults. Similarly, malnutrition does not seem to have affected the results of phenotyping numerous series of tuberculous patients. ${ }^{15}$

Complete Freund's adjuvant and hydrocortisone administration result in 'induction' of acetylation of sulphadimidine (syn:sulfamethazine) in the rabbit. ${ }^{16}$ It is not clear whether this effect is mediated via the reticuloendothelial system or via hepatocytes. The effects of such processes on phenotyping human patients is at present unknown.

Considering neoplastic disease, Bulovskaya et $\mathrm{al}^{\mathbf{1 7}}$ describe not only an increased frequency of the rapid acetylator phenotype, but also increased acetylating activity within both phenotypes in carcinoma of the breast. The present author knows of no other published series of acetylator phenotyped breast cancer patients. Lavigne et al ${ }^{18}$ report an increased acetylation of para-amino-salicylate (PAS) in patients with lymphosarcoma and acute and chronic leukaemia. It is known that PAS is monomorphically and not polymorphically acetylated in man. Nevertheless, the possibility exists that there may be a general enhancement of acetylating capability in some neoplastic disorders. It is clear, 
however, that the presence of a bronchial carcinoma does not affect the phenotyping test result (unpublished results).

(6) Satisfactory control series for comparison Satisfactory control series are required (as mentioned above) before any association between phenotype and disorder can be assessed. This is probably the most difficult point of all. It is generally considered that unaffected sibs constitute the most satisfactory control group for studies of associations between disorders and genetic phenotypes. ${ }^{19}$ Other possible controls which may be used are unrelated domiciliary controls $^{20}$ or general population controls. Using other patients who do not suffer from the disease being investigated is generally not considered a good idea because their afflictions may have some relationship to the polymorphism in question. A considerable body of data is required to rule out such a possibility.

\section{(7) Reproducibility of the statistical association}

Because of the difficulties outlined above, credence increases when similar results have been obtained by different investigators in widely different locations. For example, the highly significant association between bladder cancer and slow acetylator status shows no statistical heterogeneity between five series. ${ }^{21}$

\section{Results}

There is a clear cut association between the slow acetylator phenotype and presence of bladder cancer. This phenotype is $39 \%$ more frequently associated with this disease than it should be by chance. There is no heterogeneity between different series (table 1).

In one series, ${ }^{28}$ studied with great care, there is a highly significant association between the slow acetylator phenotype and Gilbert's disease (table 2).

In a study of Graves' disease, ${ }^{29}$ it was found that the phenotype frequencies did not differ from those in the general population. However, slow acetylators did develop the disease at a significantly younger mean age than rapid acetylators $(29.5$ years as compared with $39 \cdot 5$ years).

TABLE 2 The acetylator phenotypes in Gilbert's syndrome. ${ }^{28}$

\begin{tabular}{lllr}
\hline & $\begin{array}{l}\text { Slow } \\
\text { acetylator }\end{array}$ & $\begin{array}{l}\text { Fast } \\
\text { acetylator }\end{array}$ & Total \\
\hline Reference population & 39 & 37 & 76 \\
Gilbert's syndrome & 21 & 6 & 27 \\
Total & 60 & 43 & 103 \\
\hline
\end{tabular}

$\chi_{1}^{2}=5 \cdot 73, \mathrm{p}<0 \cdot 02$.

TABLE 3 Breast cancer data. ${ }^{17}$

\begin{tabular}{llll}
\hline & $\begin{array}{l}\text { Slow } \\
\text { acetylators }\end{array}$ & $\begin{array}{l}\text { Rapid } \\
\text { acetylatcrs }\end{array}$ & Tctal \\
\hline Breast cancer & 13 & 28 & 41 \\
Controls & 24 & 14 & 38 \\
Total & 37 & 42 & 79 \\
\hline
\end{tabular}

${ }_{1}^{2}=7 \cdot 83, \mathrm{p}<0.01$.

TABLE 1 Test of association between bladder cancer and acetylator phenotype. Modification by Haldane $e^{22}$ of the method of Woolf. ${ }^{23}$

\begin{tabular}{|c|c|c|c|c|c|c|c|c|c|}
\hline & \multicolumn{4}{|c|}{ No of subjects } & \multirow{3}{*}{$\begin{array}{l}\text { Approximate } \\
\text { relative } \\
\text { risk } x\end{array}$} & \multirow{3}{*}{$\begin{array}{l}\log _{e} e^{x} \\
=y\end{array}$} & \multirow{3}{*}{$\begin{array}{l}\text { Sampling } \\
\text { variance } \\
V\end{array}$} & \multirow{3}{*}{$\begin{array}{l}\text { Weight } \\
\frac{l}{V}=w\end{array}$} & \multirow{3}{*}{$\begin{array}{l}\text { Significance } \\
\text { of difference } \\
\text { from zero } \\
w y^{2}\end{array}$} \\
\hline & \multicolumn{2}{|c|}{ Bladder cancer } & \multicolumn{2}{|c|}{ Controls } & & & & & \\
\hline & Slow & Rapid & Slow & Rapid & & & & & \\
\hline Lower et al ${ }^{24}$ Danish* & 46 & 25 & 38 & 36 & $1 \cdot 7288$ & 0.5474 & 0.1124 & $8 \cdot 8963$ & 2.6657 \\
\hline Lower et al 24 Swedish* & 80 & 35 & 79 & 39 & $1 \cdot 1267$ & 0.1193 & 0.0776 & $12 \cdot 8827$ & 0.1834 \\
\hline Cartwright et al $25+$ & 74 & 37 & 118 & 89 & 1.4921 & 0.4002 & 0.0592 & 16.9016 & $2 \cdot 7054$ \\
\hline Woodhouse et al $26+$ & 21 & 9 & 16 & 13 & $1 \cdot 8517$ & 0.6161 & 0.2757 & 3.6270 & $1 \cdot 3767$ \\
\hline Liverpool series 21 & 66 & 34 & $510 \S$ & 342 & $1 \cdot 2932$ & 0.2571 & 0.0484 & 10.6748 & $1 \cdot 3666$ \\
\hline
\end{tabular}

Weighted mean value of $y=Y=\frac{\sum w y}{\Sigma w}-\frac{20 \cdot 7208}{62 \cdot 9824}=0.3290$.

$\mathrm{SE}$ of $\mathrm{Y}=(\mathrm{Lw})^{-\frac{1}{2}}=0 \cdot 1260$.

$95 \%$ fiduciallimits of $Y=Y \pm t_{4} 0.05(\Sigma w)^{-1}=Y \pm(2.78 \times 0.126)=0.6793$ and -0.0213 .

Antilog of $Y=X$ combined estimate of values of $X=1.389$.

The equivalent $X$ values to the $95 \%$ fiducial limts of $Y$ are 1.9725 and 0.9789

Significance of difference of $X$ from unity $=\chi_{1}^{2}=\frac{(\Sigma w y)^{2}}{\Sigma w}-6.8170(p<0.01)$.

Heterogeneity expression is tested by $x^{2}-1: \sum w y^{2}-\frac{(\Sigma w y)^{2}}{\Sigma w}=1 \cdot 4824(p>0 \cdot 10)$.

* Technique of phenotyping: Weber and Brenner. $27+$ Technique of phenotyping: monoacetyl dapsone/dapsone ratio in plasma.

$\ddagger$ Technique of phenotyping: plasma isoniazid half life. §Pooled Liverpool control data: see first part of table 5 .

From: Evans et al.2 
A surprising finding ${ }^{17}$ was both an increased frequency of the rapid acetylator phenotype and also an increased acetylating capacity within both phenotypes of patients with breast cancer (table 3 ).

Following an initial small study by Mattila and Tiitinen, ${ }^{30}$ subsequent reports show an association of the rapid acetylator phenotype with diabetes mellitus (see tables 4,5 , and 6). The criticism could be made that diabetics of different types are pooled in table 4 . Unfortunately, each UK diabetic series was not accompanied by its own control series, and so a Woolf ${ }^{23} /$ Haldane $^{22}$ analysis is only possible for diabetes using pooled UK data.

Shenfield et $a^{34}$ suggest the possibility that a high

TABLE 4 Diabetes mellitus.

\begin{tabular}{|c|c|c|c|c|c|c|c|c|c|c|}
\hline Reference & Type of diabetes & $\begin{array}{l}\text { Location } \\
\text { where study } \\
\text { conducted }\end{array}$ & Ethnic group & $\begin{array}{l}\text { Technique } \\
\text { of } \\
\text { phenotyping }\end{array}$ & $\begin{array}{l}\text { Total No } \\
\text { of patients }\end{array}$ & $\begin{array}{l}\text { No of } \\
\text { slow } \\
\text { acetylators }\end{array}$ & $q$ & $S E(q)$ & $\begin{array}{l}\text { Upper } \\
\text { confidence } \\
\text { limit }\end{array}$ & $\begin{array}{l}\text { Lower } \\
\text { confidence } \\
\text { limit }\end{array}$ \\
\hline \multirow[t]{2}{*}{30} & Juvenile & Helsinki & Finns & $\begin{array}{l}\text { Serum } \\
\text { isoniazid } \\
\text { half life }\end{array}$ & 9 & 2 & 0.4714 & $0 \cdot 1470$ & $0 \cdot 7654$ & $0 \cdot 1744$ \\
\hline & Adult & & , & , & 19 & 13 & 0.8272 & $0 \cdot 0645$ & 0.9561 & 0.6982 \\
\hline \multirow[t]{2}{*}{31} & $\begin{array}{l}\text { Random outpatient } \\
\text { clinic patients }\end{array}$ & Oxford & Not stated & 5 & 47 & 18 & 0.6189 & 0.0573 & 0.7334 & $0 \cdot 5043$ \\
\hline & $\begin{array}{l}\text { Inpatients, poor } \\
\text { control, "many } \\
\text { juvenile diabetics" }\end{array}$ & " & , & , & 38 & 19 & 0.7071 & 0.0574 & 0.8218 & 0.5924 \\
\hline \multirow[t]{2}{*}{32} & Juvenile & $\begin{array}{l}\text { Sheffield, } \\
\text { Leicester }\end{array}$ & 'Whites' & 5 & 49 & 22 & 0.6701 & 0.0530 & 0.7761 & $0 \cdot 5640$ \\
\hline & Maturity onset & , & , & & 81 & 39 & 0.6937 & $0 \cdot 0400$ & 0.7739 & 0.6139 \\
\hline \multirow[t]{2}{*}{33} & $\begin{array}{l}\text { Insulin dependent } \\
\text { without } \\
\text { complications }\end{array}$ & $\begin{array}{l}\text { South-east } \\
\text { England }\end{array}$ & Caucasian & 5 & 18 & 10 & 0.7454 & $0 \cdot 0786$ & 0.9025 & $0 \cdot 5882$ \\
\hline & $\begin{array}{l}\text { Insulin dependent } \\
\text { with complications }\end{array}$ & $"$ & ", & $"$ & 37 & 18 & 0.6975 & 0.0589 & $0 \cdot 8153$ & 0.5797 \\
\hline \multirow[t]{3}{*}{34} & Type 1 & Perth, & Causasoids & 36 & 47 & 12 & 0.5053 & $0 \cdot 0629$ & 0.6312 & $0 \cdot 3794$ \\
\hline & Type 2 & W Australia & of Europear & & 69 & 27 & $0 \cdot 6255$ & $0 \cdot 0470$ & 0.7195 & 0.5316 \\
\hline & $\begin{array}{l}\text { Normal control } \\
\text { subjects }\end{array}$ & & descent & & 112 & 58 & $0 \cdot 7196$ & $0 \cdot 0328$ & 0.7852 & $0 \cdot 6540$ \\
\hline \multirow[t]{3}{*}{35} & Juvenile onset & Madrid & Presumably & 5 & 32 & 17 & 0.7289 & $0 \cdot 0605$ & $0 \cdot 8499$ & 0.6078 \\
\hline & Maturity onset & & Spanish & & 87 & 50 & 0.7581 & 0.0350 & 0.8281 & 0.6882 \\
\hline & Controls & & Caucasians & & 157 & 90 & 0.7571 & $0 \cdot 0261$ & $0 \cdot 8093$ & $0 \cdot 7050$ \\
\hline
\end{tabular}

TABLE 5 Details of UK control series (all Caucasian).

\begin{tabular}{|c|c|c|c|c|c|c|c|c|}
\hline Reference & Health status & Technique of phenotyping & $\begin{array}{l}\text { Total No of } \\
\text { subjects }\end{array}$ & $\begin{array}{l}\text { No of slow } \\
\text { acetylators }\end{array}$ & $q$ & $S E(q)$ & $\begin{array}{l}\text { Upper } \\
\text { confidence } \\
\text { limit }\end{array}$ & $\begin{array}{l}\text { Lower } \\
\text { confidence } \\
\text { limit }\end{array}$ \\
\hline \multicolumn{9}{|c|}{ Liverpool series } \\
\hline 37 & Healthy & $\begin{array}{l}\% \text { urinary sulphamethazine } \\
\text { acetylated }\end{array}$ & 58 & 29 & 0.7071 & $0 \cdot 0464$ & $0 \cdot 8000$ & $0 \cdot 6143$ \\
\hline 38 & Tuberculous & INH T 1 & 101 & 67 & $0 \cdot 8145$ & $0 \cdot 0289$ & $0 \cdot 8722$ & 0.7567 \\
\hline 39 & Healthy & $\begin{array}{l}\% \text { urinary sulphamethazine } \\
\text { acetylated }\end{array}$ & 119 & 62 & 0.7218 & $0 \cdot 0317$ & 0.7853 & 0.6584 \\
\hline 40 & Non-schizophrenic & , & 122 & 71 & $0 \cdot 7628$ & 0.0293 & 0.8214 & 0.7043 \\
\hline 5 & $\begin{array}{l}\text { Healthy }+ \\
\text { tuberculous }\end{array}$ & $\begin{array}{l}\% \text { sulphamethazine acetylated in } \\
\text { serum and urine }\end{array}$ & 135 & 84 & $0 \cdot 7888$ & 0.0264 & $0 \cdot 8417$ & 0.7359 \\
\hline 41 & Tuberculous & ,. & 95 & 63 & $0 \cdot 8143$ & 0.0298 & 0.8739 & 0.7548 \\
\hline 42 & Healthy & , & 44 & 26 & $0 \cdot 7687$ & 0.0482 & 0.8651 & 0.6723 \\
\hline 43 & Healthy & $\begin{array}{l}\% \text { acetylation of sulphapyridine a } \\
\text { sulphamethazine in serum and } u\end{array}$ & d 50 & 28 & $0 \cdot 7483$ & $0 \cdot 0469$ & $0 \cdot 8421$ & 0.6545 \\
\hline 44 & Healthy & 5, method 2 & 27 & 15 & $0 \cdot 7454$ & 0.0642 & 0.8737 & 0.6171 \\
\hline 45 & Healthy & $\begin{array}{l}\text { Serum and urine acetylsulpha- } \\
\text { pyridine: total sulphapyridine }\end{array}$ & 26 & 17 & $0 \cdot 8086$ & 0.0577 & 0.9240 & 0.6932 \\
\hline 76 & Healthy & $\begin{array}{l}\text { Ratio of acetylisoniazid: } \\
\text { isoniazid in urine and plasma }\end{array}$ & 49 & 30 & $0 \cdot 7825$ & $0 \cdot 0445$ & $0 \cdot 8714$ & 0.6935 \\
\hline 47 & Healthy & 5, method 2 & 26 & 18 & $0 \cdot 8321$ & $0 \cdot 0544$ & 0.9408 & $0 \cdot 7233$ \\
\hline Total & & & 852 & 510 & $0 \cdot 7737$ & $0 \cdot 0109$ & $0 \cdot 7954$ & $0 \cdot 7520$ \\
\hline \multicolumn{9}{|c|}{ Non-Liverpool UK controls (additional data to form a UK control series) } \\
\hline 7 & Healthy & $\begin{array}{l}\% \text { sulphamethazine acetylated in } \\
\text { blood and urine }\end{array}$ & 25 & 14 & $0 \cdot 7843$ & $0 \cdot 0663$ & $0 \cdot 8810$ & 0.6157 \\
\hline 4 & Healthy & $\begin{array}{l}\% \text { urinary sulphamethazine } \\
\text { acetylated }\end{array}$ & 25 & 16 & $0 \cdot 8000$ & $0 \cdot 0600$ & $0 \cdot 9200$ & $0 \cdot 6800$ \\
\hline 26 & Healthy & INH T! & 29 & 16 & $0 \cdot 7428$ & $0 \cdot 0622$ & $0 \cdot 8671$ & $0 \cdot 6185$ \\
\hline \multicolumn{3}{|c|}{ Grand total } & 931 & 556 & 0.7728 & 0.0104 & 0.7936 & 0.7520 \\
\hline
\end{tabular}

INH T $\mathrm{T}_{2}^{1}=$ plasma isoniazid half life. 
TABLE 6 Test of association between diabetes and acetylator phenotype. Modification by Haldane $e^{22}$ of the method of Woolf. ${ }^{23}$

\begin{tabular}{|c|c|c|c|c|c|c|c|c|c|}
\hline \multirow[t]{3}{*}{ Source } & \multicolumn{4}{|c|}{ No of subjects } & \multirow{3}{*}{$\begin{array}{l}\text { Relative } \\
\text { incidence } \\
x\end{array}$} & \multirow{3}{*}{$\log _{y} e^{x}$} & \multirow{3}{*}{$\begin{array}{l}\text { Sampling } \\
\text { variance }\end{array}$} & \multirow{3}{*}{$\begin{array}{l}\text { Weight } \\
\frac{1}{V}=w\end{array}$} & \multirow{3}{*}{$\begin{array}{l}\text { Significance of } \\
\text { difference from } \\
\text { zero } w y^{2}\end{array}$} \\
\hline & \multicolumn{2}{|c|}{ Diabetes } & \multicolumn{2}{|c|}{ Controls } & & & & & \\
\hline & Slow & Rapid & Slow & Rapid & & & & & \\
\hline $\begin{array}{l}\text { Pooled UK data } \\
34 \\
35\end{array}$ & $\begin{array}{r}126 \\
39 \\
67\end{array}$ & $\begin{array}{r}144 \\
77 \\
52\end{array}$ & $\begin{array}{r}556 \\
58 \\
90\end{array}$ & $\begin{array}{r}375 \\
54 \\
67\end{array}$ & $\begin{array}{l}1 \cdot 6929 \\
2 \cdot 1060 \\
1 \cdot 0428\end{array}$ & $\begin{array}{l}0.5264 \\
0.7448 \\
0.0419\end{array}$ & $\begin{array}{l}0.0192 \\
0.0730 \\
0.0593\end{array}$ & $\begin{array}{l}52 \cdot 0143 \\
13 \cdot 7077 \\
16 \cdot 8723\end{array}$ & $\begin{array}{r}14.4155 \\
7 \cdot 6041 \\
0.0296\end{array}$ \\
\hline
\end{tabular}

Weighted mean value of $y=Y=\frac{\Sigma w y}{\Sigma w}=\frac{38 \cdot 2994}{82 \cdot 5944}=0.4637$.

$\mathrm{SD}$ of $\mathrm{Y}=(\Sigma \mathrm{w})^{-\frac{1}{2}}=0 \cdot 1100$

$95 \%$ fiducial limits $=Y \pm 1,0.025,2(\Sigma w)^{-\frac{1}{2}}=-0.0098$ and 0.9372 .

Antilog $\mathrm{Y}=\mathrm{X}=1 \cdot 5900$.

The equivalent $X$ values to the $95 \%$ fiducial limits of $Y$ are 0.9905 and 2.545 .

Significance of the difference of $X$ from unity $=\chi_{i}^{\prime}=\frac{(\Sigma w y)^{2}}{\Sigma w}=17.7596$.

Homogeneity estimate $=\chi_{N-1}^{2}=\Sigma w y^{2}-\frac{(\Sigma w y)^{2}}{\Sigma w}=22 \cdot 0493-17 \cdot 7596=4 \cdot 2897$.

TABLE 7 Miscellaneous disorders.

\begin{tabular}{|c|c|c|c|c|c|c|c|c|c|c|c|}
\hline Reference & Disease category & $\begin{array}{l}\text { Locations } \\
\text { survey } \\
\text { conducted }\end{array}$ & $\begin{array}{l}\text { Ethnic } \\
\text { group }\end{array}$ & $\begin{array}{l}\text { Technique of } \\
\text { phenotyping }\end{array}$ & $\begin{array}{l}\text { No of } \\
\text { patients }\end{array}$ & $\begin{array}{l}\text { No of } \\
\text { slow } \\
\text { acetylators }\end{array}$ & $q$ & $S E(q)$ & $\begin{array}{l}\text { Upper } \\
\text { confidence } \\
\text { limit }\end{array}$ & $\begin{array}{l}\text { Lower } \\
\text { confidence } \\
\text { limit }\end{array}$ & $\stackrel{\infty}{+\infty}$ \\
\hline \multicolumn{11}{|c|}{ Mental disorders } & \\
\hline 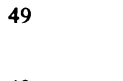 & $\begin{array}{l}\text { Chronic } \\
\text { schizophrenics on } \\
\text { neuroleptics }\end{array}$ & Helsinki & Finns & INH T $\frac{1}{2}$ & 29 & 21 & 0.851 & 0.049 & 0.948 & 0.753 & 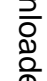 \\
\hline 40 & Schizophrenic & Liverpool & $\begin{array}{l}\text { British } \\
\text { whites }\end{array}$ & $\begin{array}{l}\% \text { urinary } \\
\text { sulphadimidine } \\
\text { acetylated }\end{array}$ & 78 & 51 & 0.809 & 0.033 & 0.875 & 0.742 & อ̆ \\
\hline \multirow[t]{2}{*}{50} & Neurotic depression & Newcastle & $\begin{array}{l}\text { British } \\
\text { whites }\end{array}$ & INH T $\frac{1}{2}$ & 25 & 16 & 0.8000 & 0.0600 & 0.9200 & 0.6800 & 3 \\
\hline & $\begin{array}{r}\text { Endogenous } \\
\text { depression }\end{array}$ & , & , & ", & 25 & 17 & 0.8246 & 0.0566 & 0.9378 & 0.7115 & $\frac{\pi}{5}$ \\
\hline \multirow[t]{3}{*}{51} & Neurotic depression & ", & ", & 36 & 29 & 19 & 0.809 & 0.055 & 0.918 & $0 \cdot 700$ & \\
\hline & $\begin{array}{l}\text { Neurotic anxiety } \\
\text { states }\end{array}$ & ", & $"$ & , & 28 & 17 & 0.779 & 0.059 & 0.898 & 0.661 & હَ' \\
\hline & Phobic anxiety states , & & & & 23 & 15 & $\begin{array}{l}0.808 \\
0.6667\end{array}$ & $\begin{array}{l}0.061 \\
0.0717\end{array}$ & $\begin{array}{l}0.931 \\
0.8101\end{array}$ & $\begin{array}{l}0.685 \\
0.523\end{array}$ & 宁 \\
\hline 52 & Neurotic depression & Glasgow & $\begin{array}{l}\text { Pre- } \\
\text { sumably } \\
\text { British } \\
\text { whites }\end{array}$ & 5 & 27 & 12 & & & & & $\begin{array}{l}3.3 . \\
\text { 을. }\end{array}$ \\
\hline 53 & Neurotic depression & Glasgow & $\begin{array}{l}\text { British } \\
\text { whites }\end{array}$ & 5 & 97 & 51 & 0.725 & 0.034 & 0.795 & 0.655 & $P$ \\
\hline 54 & Mongolism & Helsinki & Finns & INH $T \frac{1}{2}$ & 61 & 41 & 0.820 & 0.037 & 0.893 & 0.747 & \\
\hline \multicolumn{12}{|c|}{ Gastrointestinal disorders } \\
\hline 55 & Duodenal ulcer & Liverpool & $\begin{array}{l}\text { British } \\
\text { whites }\end{array}$ & 37 & 50 & 28 & 0.748 & 0.046 & $0 \cdot 842$ & 0.655 & హి \\
\hline 56 & $\begin{array}{l}\text { Postgastrectomy } \\
\text { syndrome }\end{array}$ & $\begin{array}{l}\text { Oulu and } \\
\text { Helsinki }\end{array}$ & Finns & INH $T \frac{1}{2}$ & 13 & 8 & 0.784 & 0.086 & 0.956 & 0.612 & \\
\hline 45 & $\begin{array}{l}\text { Ulcerative colitis (17), } \\
\text { Crohn's disease of } \\
\text { ileum and colon (2) }\end{array}$ & Liverpool & $\begin{array}{l}\text { British } \\
\text { whites }\end{array}$ & $\begin{array}{l}\text { Serum and urine } \\
\text { acetylsulphapyri- } \\
\text { dine: total } \\
\text { sulphapyridine }\end{array}$ & 19 & 11 & 0.7609 & 0.0744 & 0.9098 & 0.6120 & $\underset{\sigma}{\sigma}$ \\
\hline 57 & $\begin{array}{l}\text { Ulcerative colitis (84), } \\
\text { Crohn's disease (38) }\end{array}$ & Edinburgh & $\begin{array}{l}\text { British } \\
\text { whites }\end{array}$ & $\begin{array}{l}5 \text { and salicyl-azo- } \\
\text { sulphapyridine } \\
\text { metabolites }\end{array}$ & 122 & 87 & 0.844 & 0.024 & 0.893 & 0.796 & $\frac{\mathscr{C}}{\stackrel{C}{\mathbb{C}}}$ \\
\hline 58 & $\begin{array}{l}\text { Crohn's disease (14), } \\
\text { ulcerative colitis } \\
\text { (10), undifferentiated } \\
\text { CIBD (4) }\end{array}$ & $\begin{array}{l}\text { Saskatoon, } \\
\text { Saskat- } \\
\text { d chewan }\end{array}$ & Canadian & $\begin{array}{l}\text { Serum ratio } \\
\text { acetylsulpha- } \\
\text { pyridine: total } \\
\text { sulphapyridine }\end{array}$ & 28 & 19 & 0.8238 & 0.0536 & 0.9309 & 0.7166 & $\stackrel{0}{\stackrel{0}{D}}$ \\
\hline 59 & $\begin{array}{l}\text { Ulcerative colitis (20), } \\
\text { Crohn's disease (25) }\end{array}$ & $\begin{array}{l}\text { Buffalo, } \\
\text { NY }\end{array}$ & Not stated & $\begin{array}{l}\text { Sulphasalazine } \\
\text { metabolism as giver } \\
\text { in ref } 57\end{array}$ & $\operatorname{len}^{45}$ & 28 & $0 \cdot 7888$ & 0.0458 & 0.8804 & 0.6972 & $\frac{\rho}{\mathbb{D}}$ \\
\hline
\end{tabular}


TABLE 7-continued.

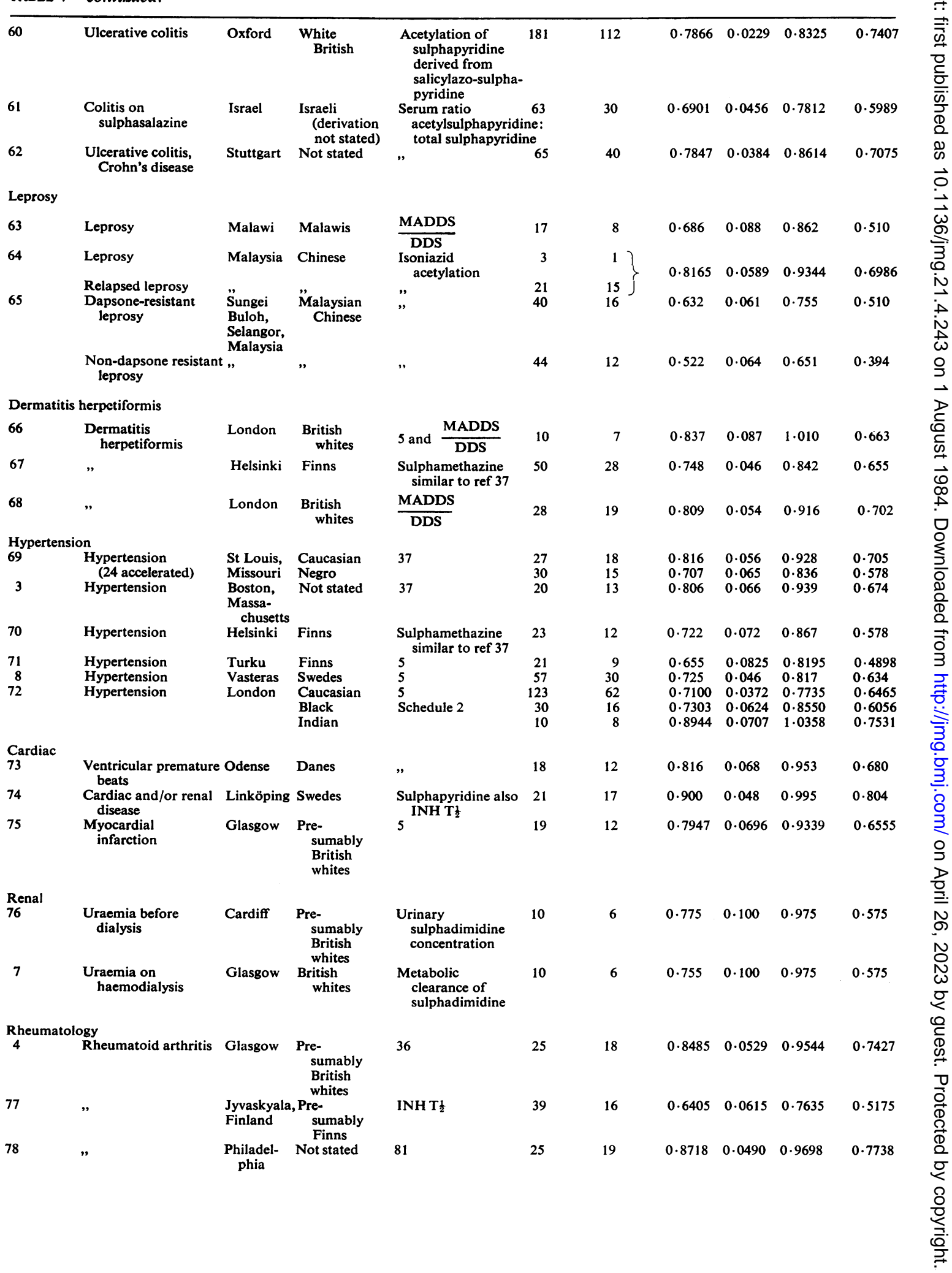


TABLE 7-continued.

\begin{tabular}{|c|c|c|c|c|c|c|c|c|c|c|}
\hline 79 & , & Jerusalem & $\begin{array}{l}\text { Israelis } \\
\text { of hetero- } \\
\text { geneous } \\
\text { origin }\end{array}$ & $\frac{\text { MADDS }}{\text { DDS }}$ & 43 & 28 & 0.8069 & 0.0450 & 0.8969 & 0.7169 \\
\hline $\begin{array}{l}\text { Hepatic } \\
80\end{array}$ & $\begin{array}{l}\text { Porphyria cutanea } \\
\text { tarda }\end{array}$ & Madrid & $\begin{array}{l}\text { Pre- } \\
\text { sumably } \\
\text { Spanish }\end{array}$ & 5 & 51 & 33 & 0.8044 & 0.0416 & 0.8876 & 0.7212 \\
\hline
\end{tabular}

INH T $\frac{1}{2}=$ plasma isoniazid half life.

$\frac{\text { MADDS }}{\text { DDS }}=$ ratio $\frac{\text { mono acetyl diamino diphenyl sulphone }}{\text { diamino diphenyl sulphone }}$ in urine.

CIBD chronic inflammatory bowel disease.

TABLE 8 Chinese control subjects.

\begin{tabular}{|c|c|c|c|c|c|c|c|c|c|}
\hline Reference & $\begin{array}{l}\text { Disease } \\
\text { category }\end{array}$ & $\begin{array}{l}\text { Location } \\
\text { survey } \\
\text { conducted }\end{array}$ & Technique of phenotyping & $\begin{array}{l}\text { No of } \\
\text { subjects }\end{array}$ & $\begin{array}{l}\text { No of } \\
\text { slow } \\
\text { acetylators }\end{array}$ & $q$ & $S E(q)$ & $\begin{array}{l}\text { Upper } \\
\text { confidence } \\
\text { limit }\end{array}$ & $\begin{array}{l}\text { Lower } \\
\text { confidence } \\
\text { limit }\end{array}$ \\
\hline $\begin{array}{l}86 \\
87 \\
88\end{array}$ & $\begin{array}{l}\text { Healthy } \\
\text { Tuberculous } \\
\text { Healthy }\end{array}$ & $\begin{array}{l}\text { Taiwan } \\
\text { Singapore, } \\
\text { Hong Kong } \\
\text { Liverpool }\end{array}$ & $\begin{array}{l}\text { Plasma INH concentration } \\
\text { Sulphadimidine and INH } \\
\text { Plasma INH concentration }\end{array}$ & $\begin{array}{r}121 \\
386 \\
184 \\
59\end{array}$ & $\begin{array}{l}27 \\
83 \\
40 \\
13\end{array}$ & $\begin{array}{l}0.4724 \\
0.4637 \\
0.4663 \\
0.4694\end{array}$ & $\begin{array}{l}0.0401 \\
0.0225 \\
0.0326 \\
0.0575\end{array}$ & $\begin{array}{l}0.5525 \\
0.5088 \\
0.5315 \\
0.5844\end{array}$ & $\begin{array}{l}0.3923 \\
0.4186 \\
0.4010 \\
0.3544\end{array}$ \\
\hline
\end{tabular}

INH = isoniazid

plasma glucose concentration causes greater acetylation in diabetics. They show neither a histogram nor a scattergram of their data. The division between the phenotypes would be expected to be blurred if variably raised plasma glucose was contributing a further component of variance to the distribution of both phenotypes. This would not appear to be so in the scattergram of Bodansky et al ${ }^{33}$ Furthermore,

TABLE 9 Acetylator phenotypes in Chinese leprosy patients and controls.

\begin{tabular}{|c|c|c|c|}
\hline & \multicolumn{2}{|c|}{ Acetylator phenotype } & \multirow[t]{2}{*}{ Total } \\
\hline & Slow & Rapid & \\
\hline $\begin{array}{l}\text { Leprosy patients } \\
\text { Control subjects }\end{array}$ & $\begin{array}{r}44 \\
163\end{array}$ & $\begin{array}{r}64 \\
587\end{array}$ & $\begin{array}{l}108 \\
750\end{array}$ \\
\hline Total & 207 & 651 & 858 \\
\hline
\end{tabular}

$\chi_{1}^{2}=17 \cdot 6$.
Mattila and Tiitinen $^{30}$ present a histogram of isoniazid half lives which show a clear bimodality, and the mean isoniazid half life in both phenotypes in diabetics closely resembled those found in nondiabetics. These findings are not at variance with those of Thom et al, ${ }^{48}$ whose experimental nondiabetic normal subjects showed shortened isoniazid half lives following large glucose doses.

For many miscellaneous conditions the data available do not suggest any significant association with either acetylator phenotype (table 7).

With regard to leprosy, there is no reliable comparison group for the Malawi patients. ${ }^{82-85}$ For the Chinese patients, the pooled estimate is $q=0.6383$, SE $(q)=0.0370$ (table 7). The three available Chinese control groups (table 8) give very consistent estimates and when they are pooled $q=0.4662$, SE (q) $=0.0162$. This suggests that in Chinese the

TABLE 10 Acetylator phenotyping of hydralazine induced systemic lupus erythematosus patients.

\begin{tabular}{|c|c|c|c|c|c|}
\hline Reference & $\begin{array}{l}\text { Location where study } \\
\text { conducted }\end{array}$ & Ethnic group & Technique of phenotyping & Total No of patients & $\begin{array}{l}\text { No of slow } \\
\text { acetylators }\end{array}$ \\
\hline $\begin{array}{l}69 \\
89 \\
90\end{array}$ & $\begin{array}{l}\text { St Louis and La Jolla } \\
\text { Stockholm and Lulea } \\
\text { London, Stoke-on-Trent, } \\
\text { and Leicester }\end{array}$ & $\begin{array}{l}\text { Caucasian } \\
\text { Presumably Caucasian } \\
\text { Presumably Caucasian }\end{array}$ & $\begin{array}{l}37 \\
\text { Isoniazid half life } \\
15\end{array}$ & $\begin{array}{l}12 \\
30^{*} \\
26\end{array}$ & $\begin{array}{l}12 \\
29 \\
25\end{array}$ \\
\hline $\begin{array}{l}72 \\
\text { and Harland SJ, } \\
\text { personal communicatio } \\
1982\end{array}$ & $\begin{array}{l}\text { London } \\
\text { on, }\end{array}$ & $\begin{array}{l}\text { Caucasian } \\
\text { Black } \\
\text { Indian }\end{array}$ & $\begin{array}{l}5 \\
\text { Method } 2\end{array}$ & $\begin{array}{r}12 \\
1 \\
-\end{array}$ & $\begin{array}{r}11 \\
1 \\
-\end{array}$ \\
\hline
\end{tabular}

*One patient of uncertain status omitted. 
TABLE 11 Systemic lupus erythematosus patients and controls.

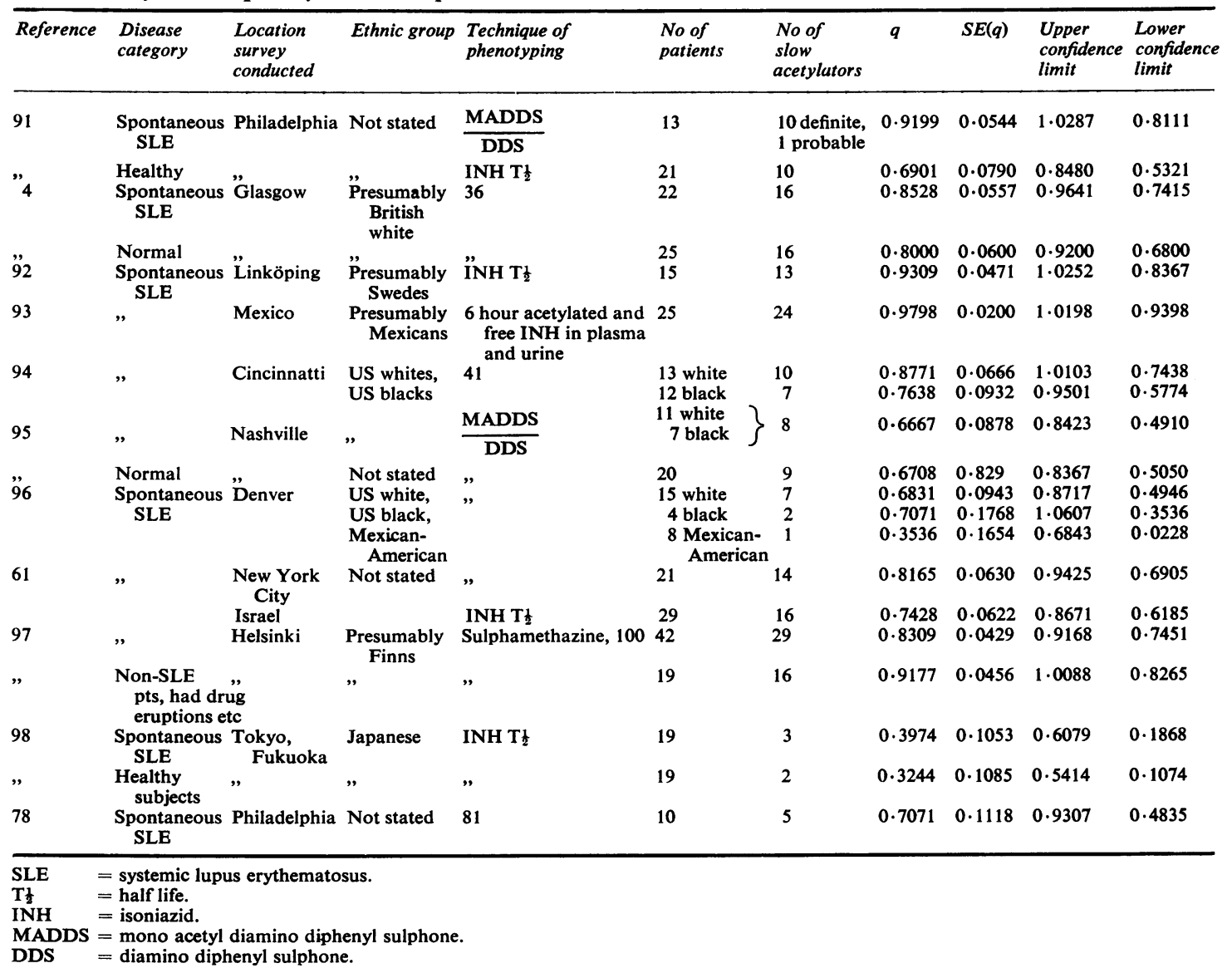

slow acetylators may be more prone than rapid acetylators to develop leprosy (table 9).

Another disorder which merits special consideration is spontaneous systemic lupus erythematosus (SLE). There is a strong association between the slow acetylator phenotype and hydralazine induced SLE (table 10). The idea was therefore promulgated that spontaneous SLE might also be associated with the slow acetylator phenotype. Sufficient information is now available from different ethnographic groups to show that this idea is not tenable (table 11).

\section{Discussion}

The interpretation of the association between bladder cancer and the slow acetylator phenotype rests upon the knowledge that carcinogenic amines are polymorphically acetylated. ${ }^{99}$ Since it is usually survivors undergoing regular cystoscopic examina- tions who are studied, the acetylator phenotype may be considered either to predispose to the development of the disease or to be a factor leading to enhanced survival (as compared with rapid acetylators). Neither the first nor the second of these explanations can be discounted on the evidence at present available.

With regard to the other associations described with (1) Gilbert's disease, (2) diabetes mellitus, (3) breast cancer, (4) leprosy, and (5) age at onset of Graves' disease, logical interpretation is much more difficult. The natural substrates for the polymorphic $\mathrm{N}$-acetyl-transferase enzyme are unknown. It may be speculated that acetyl accepting molecules, for example aromatic amines or amino-methyl-uracils, may be involved in some way in the production of these four disorders. This present survey may stimulate researchers to investigate such possibilities. 


\section{References}

1 Rabham NB, Minkin W. Criteria for classification of systemic lupus erythematosus. JAMA 1975;231:846.

2 Walstad RA. Inaktivering av medikamenter ved acetylering. Tidsskr Nor Laegeforen 1975;2:100-3.

3 Zacest R, Koch-Weser J. Relation of hydralazine plasma concentration to dosage and hypotensive action. Clin Pharmacol Ther 1972;13:420-5.

4 Lawson DH, Henry DA, Lowe J, Reavey P, Rennie JAN, Solomon A. Acetylator phenotype in spontaneous SLE and rheumatoid arthritis. Ann Rheum Dis 1979;38:171-3.

5 Evans DAP. An improved and simplified method of detecting the acetylator phenotype. J Med Genet 1969; 6:405-7.

${ }^{6}$ Godeau P, Aubert M, Imbert JC, Herreman G. Lupus erythemateux dissemine et taux d'isoniazide actif. Ann Med Interne (Paris) 1973;124:181-6.

7 Fine A, Sumner DJ. Determination of acetylator status in uraemia. Br J Clin Pharmacol 1975;2:475-6.

8 Hall S. Evaluation of the sulphadimidine acetylator phenotyping test in patients with reduced renal function. Act Med Scand 1981;209:505-7.

${ }^{9}$ Levi AJ, Sherlock S, Walker D. Phenylbutazone and isoniazid metabolism in patients with liver disease in relation to previous drug therapy. Lancet $1968 ; \mathbf{i}: 1275-9$.

10 Lester D. The acetylation of isoniazid in alcoholics. Q J Stud Alc 1965;25:541-3.

11 Olsen $\mathrm{H}$, Morland J. Ethanol-induced increase in drug acetylation in man and isolated rat cells. $B r$ Med $J 1978$; ii : $1260-2$.

12 Olsen H, Morland J. Ethanol-induced increase in procainamide acetylation in man. Br J Clin Pharmacol 1982; 13:203-8.

13 Shastri RA, Krishnaswamy K. Metabolism of sulphadiazine in malnutrition. Br J Clin Pharmacol 1979;7:69-73.

14 Shastri RA. Undernourished adults and acetylation phenotype. Int J Clin Pharmacol Ther Toxicol 1982;20: 194-6.

15 Viznerova A, Sladikova Z, Ellard GA. The determination of the acetylator phenotype of tuberculosis patients in Czechoslovakia using sulphadimidine. Tubercle 1973;54: 67-71.

16 du Souichi P, Courteau H. Induction of acetylating capacity with complete Freund's adjuvant and hydrocortisone in the rabbit. Drug Metab Dispos 1981 ;9:279-83.

17 Bulovskaya LN, Krupkin RG, Bochina TA, Shipkova AA, Pavlova MV. Acetylator phenotype in patients with breast cancer. Oncology 1978;35:185-8.

18 Lavigne JG, Barry A, d'Auteuil C, Delage JM. p-aminosalicylate metabolism in cancer patients sensitive and resistant to chemotherapy. Br J Cancer 1977;35:580-6.

19 Clarke CA, Edwards JWL, Haddock DRW, Howel-Evans AW, McConnell RB. ABO blood groups and secretor character in duodenal ulcer-population and sibship studies. Br Med J 1956;ii:725-31.

20 Evans DAP, Donohoe WTA, Hewitt S, Linaker BD. Le blood group substance degradation in the human alimentary tract and urinary $\mathrm{Le}^{\mathrm{a}}$ in coeliac disease. Vox Sang $1982 ; 43: 177-87$

21 Evans DAP, Eze LC, Whibley EJ. The association of the slow acetylator phenotype with bladder cancer. $J$ Med Genet $1983 ; 20: 330-3$.

22 Haldane JBS. The estimation and significance of the logarithm of a ratio of frequence. Ann Hum Genet 1955; 20:309-11.

23 Woolf $\mathrm{B}$. On estimating the relation between blood group and disease. Ann Hum Genet 1954:19:251-3.

24 Lower GM Jr, Nilsson T, Neson CE, Wolf H, Gamsky TE, Bryan GT. N-acetyltransferase phenotype and risk in urinary bladder cancer: approaches in molecular epidemiology. Preliminary results in Sweden and Denmark. Environ Health Perspect 1979;29:71-9.

25 Cartwright RA, Rogers HJ, Barham-Hall D, et al. Role of $\mathrm{N}$-acetyltransferase phenotypes in bladder carcinogenesis: a pharmacogenetic epidemiological approach to bladder cancer. Lancet 1982; ii:842-6.

26 Woodhouse KW, Adams PC, Clothier A, Mucklow JC, Rawlins MD. N-acetylation phenotype in bladder cancer. Hum Toxicol $1982 ; 1: 443-5$.

27 Weber WW, Brenner W. A filter paper method for determining isoniazid acetylator phenotype. Am J Hum Genet 1974:26:467-73.

28 Platzer R, Kupfer A, Bircher J, Preisig R. Polymorphic acetylation and aminopyrine demethylation in Gilbert's syndrome. Eur J Clin Invest 1978;8:219-23.

29 Ladero JM, Cano F. Fenotipo acetilador en la enfermedad de Basedow. N Arch Fac Med $1983 ; 41: 79-81$.

30 Mattila MJ, Tiitinen $\mathrm{H}$. The rate of isoniazid inactivation in Finnish diabetic and non-diabetic patients. Ann Med Exp Biol Fenn 1967;45:423-7.

31 Burrows AW, Hockaday TDR, Mann JI, Taylor JG. Diabetic dimorphism according to acetylator status. $\mathrm{Br}$ Med J 1978;i:208-10.

32 McLaren EH, Burden AC, Moorhead PJ. Acetylator phenotype in diabetic neuropathy. $B r$ Med J 1977;ii: 291-3.

33 Bodansky HJ, Drury PL, Cudworth AG, Evans DAP. Acetylator phenotypes and type I (insulin-dependent) diabetics with microvascular disease. Diabetes $1981 ; 30$ : 907-10.

34 Shenfield GM, McCann VJ, Tjokresetio R. Acetylator status and diabetic neuropathy. Diabetologia 1982;22: $441-4$.

${ }^{35}$ Ladero JM, Arrojo A, de Salamanca RE, Gomez M, Cano F, Alfonso M. Hepatic acetylator phenotype in diabetes mellitus. Ann Clin Res 1982;14:187-9.

36 Schröder H. Simplified method for determining acetylator phenotype. Br Med J 1972;3:506-7.

37 Evans DAP, White TA. Human acetylation polymorphism. J Lab Clin Med 1964;63:394-403.

38 Gow JG, Evans DAP. A study of the influence of the isoniazid inactivator phenotype on reversion in genitourinary tuberculosis. Tubercle $1964 ; 45$ : 136-43.

39 White TA, Evans DAP. The acetylation of sulfamethazine and sulfamethoxypyridazine by human subjects. Clin Pharmacol Ther 1968;9:80-8.

${ }^{40}$ Price J. Demethylation, methylation and schizophrenia. A pharmacogenetic study. MD thesis, University of London, 1971.

41 Evans DAP, Bullen MG, Houston J, Hopkins CA, Vetters JM. Antinuclear factor in rapid and slow acetylator patients treated with isoniazid. J Med Genet 1972;9: 53-6.

42 Eze LC, Evans DAP. The use of the Autoanalyser to determine the acetylator phenotype. J Med Genet 1972;9: 57-9.

43 Schröder H, Evans DAP. The polymorphic acetylation of sulphapyridine in man. $J$ Med Genet 1972;9:168-71.

44 Schröder H, Evans DAP. Acetylator phenotype and adverse effects of sulphasalazine in healthy subjects. Gut $1972 ; 13: 278-84$.

45 Schröder H, Lewkonia RM, Evans DAP. Metabolism of salicylazosulfapyridine in healthy subjects and in patients with ulcerative colitis. Clin Pharmacol Ther 1973;14: 802-9.

46 Ellard GA, Gammon PT, Tiitinen H. Determination of the acetylator phenotype using matrix isoniazid. Tubercle $1975 ; 56: 203-9$. 
47 Karim AKMB, Evans DAP. Polymorphic acetylation of nitrazepam. J Med Genet 1976;13:17-9.

48 Thom S, Farrow PR, Santoso B, Alberti KGMM, Rawlins MD. Effects of oral glucose on isoniazid kinetics. Br J Clin Pharmacol 1981 ;11:423P.

49 Mattila MJ, Takki S. Half-lives of isoniazid and salicylic acid in serum and their modification by different drugs in psychiatric patients. Ann Med Exp Biol Fenn1969;47:124-8.

50 Evans DAP, Davison K, Pratt RTC. The influence of acetylator phenotype on the effects of treating depression with phenelzine. Clin Pharmacol Ther 1965;6:430-5.

51 Marshall EF, Mountjoy CQ, Campbell IC, Garside RF, Leitch IM, Roth M. The influence of acetylator phenotype on the outcome of treatment with phenelzine in a clinical trial. Br J Clin Pharmacol 1978;6:247-54.

52 Caddy B, Tilstone WJ, Johnston EC. Phenelzine in urine: assay and relation to acetylator status. $\mathrm{Br} J \mathrm{Clin}$ Pharmacol 1976;3:633-7.

53 Johnstone ED, Marsh W. Acetylator status and response to phenelzine in depressed patients. Lancet $1973 ; \mathrm{i}: 567-70$.

54 Airaksinen E, Mattila MJ, Ollila $O$. Inactivation of isoniazid and sulphadimidine in Mongoloid subjects. Ann Med Exp Biol Fenn 1969;47:303-7.

55 Evans DAP. The acetylator phenotypes of duodenal ulcer patients. Scand J Gastroenterol 1967;2:289-92.

56 Mattila MJ, Frimen A, Larmi TKI, Koskinen R. Absorption of ethionamide, isoniazid and aminosalicylic acid from the post-resection gastrointestinal tract. Ann Med Exp Biol Fenn 1969;7:209-12.

57 Das KM, Eastwood MA. Acetylation polymorphism of sulfapyridine in patients with ulcerative colitis and Crohn's disease. Clin Pharmacol Ther 1975;18:514-20.

58 Sharp ME, Wallace SM, Hindmarsh KW, Brown MA. Acetylator phenotype and serum levels of sulfapyridine in patients with inflammatory bowel disease. Eur $J$ Clin Pharmacol $1981 ; 21: 243-50$.

59 Clarke DF, George D, Milsap RL, et al. Sulfasalazine metabolite pharmacokinetics in pediatric patients with inflammatory bowel disease. Pediatr Pharmacol 1982;2: 323-33.

60 Khan AKA, Nurazzaman M, Truelove SC. The effect of the acetylator phenotype on the metabolism of sulphasalazine in man.J Med Genet $1983 ; 20: 30-6$.

61 Reidenberg MM, Levy M, Drayer DR, Zylber-Katz E, Robbins WC. Acetylator phenotype in idopathic systemic lupus erythematosus. Arthritis Rheum 1980;23:569-73.

${ }^{62}$ Fisher C, Klotz U. High performance liquid chromatographic determination of aminosalicylate, sulfapyridine and their metabolites. Its application for pharmacokinetic studies with salicylazosulfapyridine in Man. J Chromatogr $1979 ; 162: 237-43$.

63 Ellard GA, Gammon PT, Harris JM. The application of urine tests to monitor the regularity of dapsone selfadministration. Lepr Rev 1974;45:224-34.

64 Ellard GA, Gammon PT, Helmy HS, Rees RJW. Dapsone acetylation and the treatment of leprosy. Nature 1972;239:159-60.

65 Gelber RH, Rees RJW. Dapsone metabolism in patients with dapsone-resistant leprosy. Am J Trop Med Hyg 1975; 24:963-7.

o6 Ellard GA, Gammon PT, Savin JA, Tan RSH. Dapsone acetylation in dermatitis herpetiformis. $\mathrm{Br} J$ Dermatol $1974 ; 90: 441-4$.

37 Forstrom L, Mattila MJ, Mustakallio KK. Acetylator phenotype, minimal maintenance dose and haemolytic effect of dapsone in dermatitis herpetiformis. Ann Clin Res 1974;6:308-10.

88 Swain AF, Ahmad RA, Rogers HJ, Leonard JN, Fry L. Pharmacokinetic observations on dapsone in dermatitis herpetiformis. BrJ Dermatol 1983 ;108:91-8.
69 Perry HM, Tan EM, Carmody S, Sakamoto A. Relationship of acetyl transferase activity to antinuclear antibodies and toxic symptoms in hypertensive patients treated with hydralazine. J Lab Clin Med 1970;76:114-25.

70 Jounela AJ, Pasanen M, Mattila MJ. Acetylator phenotype and the anti-hypertensive response to hydralazine. Acta Med Scand 1975;197:303-6.

71 Iisalo E, Laine T, Lehtonen A, Sellman R. Dihydralazine therapy and acetylator phenotype. Int J Clin Pharmacol Biopharm 1979;17:119-24.

72 Mansilla Tinoco R, Harland SJ, Ryan PJ, et al. Hydralazine, antinuclear antibodies and the lupus syndrome. Br Med J 1982;284:936-9.

${ }_{73}$ Schröder P, Klitgaard NA, Simonsen E. Significance of the acetylator phenotype and the therapeutic effect of procainamide. Eur J Clin Pharmacol 1979;15:63-8.

74 Molin L, Larsson R, Karlsson E. Evaluation of the sulphapyridine acetylator phenotyping test in healthy subjects and in patients with cardiac and renal disease. Acta Med Scand 1977;201:217-22.

75 Campbell W, Tilstone WJ, Lawson DH, Hutton I, Lawrie TDV. Acetylator phenotype and the clinical pharmacology of slow-release procainamide. Br J Clin Pharmacol 1976;3:1023-6.

76 Williams DM, Wimpenny J, Asscher AW. Renal clearance of sodium sulphadimidine in normal and uraemic subjects. Lancet 1968 ;ii:1058-60.

77 Oka M, Seppala O. Acetylation phenotype in rheumatoid arthritis. Scand J Rheumatol 1978;7:29-30.

78 Ehrlich GE, Freeman-Narrod M, Wineburgh GS. Predominance of slow acetylators among patients with rheumatoid arthritis. Eur J Rheumatol Inflam 1979;2: 196-8.

79 Ehrenfeld M, Levy M, Zylber-Katz E. Acetylator phenotype in rheumatoid arthritis. Isr J Med Sci 1983;19: 368-70.

80 Ladero JM, de Salamanca RE, Chinarro S. Hepatic acetylator phenotype in porphyria cutanea tarda. Arch Dermatol Res 1981 ;270:171-3.

81 Peters JH, Gordon GR, Karat AB. Polymorphic acetylation of the antibacterials sulfamethazine and dapsone in South Indian subjects. Am J Trop Med Hyg 1975;24: 641-8.

82 Karim AKMB, Elfellah MS, Evans DAP. Human acetylator polymorphism: estimate of allele frequency in Libya and details of global distribution. $J$ Med Genet $1981 ; 18: 325-30$.

83 Hodgkin MM, Eidus L, Bailey WC. Isoniazid phenotyping of black as well as white patients. Can J Physiol Pharmacol 1979;57:760-3.

84 Glatthaar E, Gartig D, Stander MF, Kleeberg HH. Isoniazid levels in black patients dosed with a matrix preparation (Tebesium). Prax Pneumol 1977;31:885-9.

85 Eidus L, Glatthaar E, Hodgkin MM, Nel EE, Kleeberg HH. Comparison of isoniazid phenotyping of black and white patients with emphasis on South African blacks. Int J Clin Pharmacol Biopharm 1979;17:311-6.

86 Sunahara S, Urano M, Ogawa M, Yoshida S, Mukoyama $\mathbf{H}$, Kawai K. Genetic aspect of isoniazid metabolism. Jpn J Hum Genet 1963;8:93-111.

87 Ellard GA, Gammon PT. Acetylator phenotyping of tuberculosis patients using matrix isoniazid or sulphadimidine and its prognostic significance for treatment with several intermittent isoniazid-containing regimens. $B r J$ Clin Pharmacol 1977;4:5-14.

88 Evans DAP. Pharmacogenetics. Am J Med 1963;34: 639-63.

89 Strandberg I, Boman G, Hassler L, Sjöqvist F. Acetylator phenotype in patients with hydralazine-induced lupoid syndrome. Acta Med Scand 1976;200:367-71. 
${ }^{90}$ Batchelor JR, Welsh KI, Mansilla-Tinoco $\mathrm{R}$, et al. Hydralazine-induced systemic lupus erythematosus: influence of HLA-DR and sex on susceptibility. Lancet $1980 ; \mathbf{i}: 1107-9$.

91 Reidenberg MM, Martin JH. The acetylator phenotype of patients with systemic lupus erythematosus. Drug Metab Dispos 1974;2:71-3.

92 Larsson R, Karlsson E, Molin L. Spontaneous systemic lupus erythematosus and acetylator phenotype. Acta Med Scand 1977;201:223-6.

${ }^{93}$ Fishbein E, Alarcon-Segovia D. Slow acetylation phenotype in systemic lupus erythematosus. Arthritis Rheum $1979 ; 22: 95-6$.

94 Foad B, Litwin A, Zimmer H, Hess EV. Acetylator phenotype in systemic lupus erythematosus. Arthritis Rheum 1977;20:815-8.

95 Vansant J, Woosley RL, John JT, Sergent JS. Normal distribution of acetylator phenotypes in systemic lupus erythematosus. Arthritis Rheum 1978;21:192-5.

${ }^{96}$ Morris RJ, Freed CR, Kohler PF. Drug acetylation phenotype unrelated to development of spontaneous systemic lupus erythematosus. Arthritis Rheum 1979;22: 777-80.
97 Johansson EA, Mustakallio KK, Mattila MM, Tiilkainen A. Cutaneous reactions to drugs, acetylation phenotype and HLA antigens in patients with and without systemic lupus erythematosus (SLE). Ann Clin Res 1976;8:126-8.

98 Horai Y, Ishizaki T, Sasaki T, Koya G, Matsuyama K, Iguchi S. Isoniazid disposition, comparison of isoniazid phenotyping methods in, and acetylator distribution of Japanese patients with idiopathic systemic lupus erythematosus and control subjects. Br J Clin Pharmacol 1982; 13:361-74.

99 Glowinski IB, Radtke HE, Weber WW. Genetic variation in $\mathrm{N}$-acetylation of carcinogenic arylamines by human and rabbit liver. Mol Pharmacol 1978;14:940-9.

100 Mattila MJ, Tiitinen H, Alhava E. Acetylation pattern of different sulphonamides in rapid and slow isoniazid inactivators. Ann Med Exp Biol Fenn 1969;47:308-15.

Correspondence and requests for reprints to Dr D A Price Evans, Director of Medicine, Riyadh Armed Forces Hospital, PO Box 7897, Riyadh 11159, Saudi Arabia. 